an Applied Biosystems 7700 sequence detector. Relative occupancy values were calculated by determining the apparent immunoprecipitation efficiency (amount of PCR product in the immunoprecipitated sample divided by the amount of PCR product in the input sample) and normalized to the level observed at the coding sequence of the POL1 gene, which was defined as 1 . This background binding was then subtracted to give a value in 'occupancy units'. Error bars shown reflect the standard deviation of the mean of independent experiments.

\section{Western blotting}

TAP (tandem affinity purification)-tagged Ifhl was detected with peroxidase-antiperoxidase antibody (Sigma) and Fhll was detected with anti-myc antibody (9e10, Santa Cruz Biotechnology).

\section{Microarray analysis}

Microarrays containing duplicate spots of 6,528 PCR products corresponding to nearly al yeast intergenic regions were hybridized with a mixture of amplified immunoprecipitate (labelled with Cy5 fluorescent dye) and input (labelled with Cy3 dye) samples, as described previously ${ }^{27}$. Values shown are an average of two independent experiments. Conserved DNA motifs were identified using AlignACE ${ }^{28}$. WebLogo was used to generate motif $\log ^{29}$.

\section{Transcriptional analysis}

Total RNA was purified using Qiagen RNeasy columns with DNase I treatment. Firststrand $\mathrm{CDNA}$ was synthesized using $\mathrm{dT}_{16}$, and quantitative $\mathrm{PCR}$ in real time was performed on the resulting first-strand complementary DNA using primers specific to the gene of interest $^{30}$. RNA levels were determined relative to a control gene, ACT1.

Received 7 September; accepted 5 November 2004; doi:10.1038/nature03175.

1. Mager, W. H. Control of ribosomal protein gene expression. Biochim. Biophys. Acta 949, 1-15 (1988)

2. Pogue-Geile, K. et al. Ribosomal protein genes are overexpressed in colorectal cancer: isolation of a cDNA encoding the human S3 ribosomal protein. Mol. Cell. Biol. 11, 3842-3849 (1991).

3. Seshadri, T., Uzman, J. A., Oshima, J. \& Campisi, J. Identification of a transcript that is downregulated in senescent human fibroblasts. Cloning, sequence analysis, and regulation of the human L7 ribosomal protein gene. J. Biol. Chem. 268, 18474-18480 (1993).

4. Pierandrei-Amaldi, P. \& Amaldi, F. Aspects of regulation of ribosomal protein synthesis in Xenopus laevis. Genetica 94, 181-193 (1994).

5. Warner, J. R. The economics of ribosome biosynthesis in yeast. Trends Biochem. Sci. 24, 437-440 (1999).

6. Lieb, J. D., Liu, X. L., Botstein, D. \& Brown, P. O. Promoter-specific binding of Rapl revealed by genome-wide maps of protein-DNA association. Nature Genet. 28, 327-334 (2001).

7. Moehle, C. M. \& Hinnebusch, A. G. Association of RAP1 binding sites with stringent control of ribosomal protein gene transcription in Saccharomyces cerevisiae. Mol. Cell. Biol. 11, 2723-2735 (1991).

8. Klein, C. \& Struhl, K. Protein kinase A mediates growth-regulated expression of yeast ribosomal protein genes by modulating RAP1 transcriptional activity. Mol. Cell. Biol. 14, 1920-1928 (1994).

9. Li, B., Nierras, C. R. \& Warner, J. R. Transcriptional elements involved in the repression of ribosomal protein synthesis. Mol. Cell. Biol. 19, 5393-5404 (1999).

10. Mencia, M., Moqtaderi, Z., Geisberg, J. V., Kuras, L. \& Struhl, K. Activator-specific recruitment of TFIID and regulation of ribosomal protein genes in yeast. Mol. Cell 9, 823-833 (2002).

11. Shore, D. RAP1: a protean regulator in yeast. Trends Genet. 10, 408-412 (1994).

12. Morse, R. H. RAP, RAP, open up! New wrinkles for RAP1 in yeast. Trends Genet. 16, 51-53 (2000).

13. Neuman-Silberberg, F. S., Bhattacharya, S. \& Broach, J. R. Nutrient availability and the RAS/cyclic AMP pathway both induce expression of the ribosomal protein genes in Saccharomyces cerevisiae but by different mechanisms. Mol. Cell. Biol. 15, 3187-3196 (1995).

14. Cardenas, M. E., Cutler, N. S., Lorenz, M. C., Di Como, C. J. \& Heitman, J. The TOR signaling cascade regulates gene expression in response to nutrients. Genes Dev. 13, 3271-3279 (1999).

15. Powers, T. \& Walter, P. Regulation of ribosome biogenesis by the rapamycin-sensitive TOR-signaling pathway in Saccharomyces cerevisiae. Mol. Biol. Cell 10, 987-1000 (1999).

16. Lascaris, R. F., Mager, W. H. \& Planta, R. J. DNA-binding requirements of the yeast protein Raplp as selected in silico from ribosomal protein gene promoter sequences. Bioinformatics 15, 267-277 (1999).

17. Hermann-Le Denmat, S., Werner, M., Sentenac, A. \& Thuriaux, P. Suppression of yeast RNA polymerase III mutations by FHL1, a gene coding for a fork head protein involved in rRNA processing. Mol. Cell. Biol. 14, 2905-2913 (1994).

18. Lee, T. I. et al. Transcriptional regulatory networks in Saccharomyces cerevisiae. Science 298, 799-804 (2002).

19. Cherel, I. \& Thuriaux, P. The IFHI gene product interacts with a forkhead protein in Saccharaomyce cerevisiae. Yeast 11, 261-270 (1995).

20. Goncalves, P. M. et al. Transcription activation of yeast ribosomal protein genes requires additional elements apart from binding sites for Abflp or Raplp. Nucleic Acids Res. 23, 1475-1480 (1995).

21. Beer, M. A. \& Tavazoie, S. Predicting gene expression from sequence. Cell 117, 185-198 (2004).

22. Kim, M., Ahn, J. W., Song, K., Pack, K. H. \& Pai, H. S. Forkhead-associated domains of the tobacco NtFHAl transcription activator and the yeast Fhll forkhead transcription factor are functionally conserved. J. Biol. Chem. 277, 38781-38790 (2002).

23. Brachmann, C. B. et al. Designer deletion strains derived from Saccharomyces cerevisiae S288C: a useful set of strains and plasmids for PCR-mediated gene disruption and other applications. Yeast 14 115-132 (1993).

24. Cosma, M. P., Tanaka, T. \& Nasmyth, K. Ordered recruitment of transcription and chromatin remodeling factors to a cell cycle- and developmentally regulated promoter. Cell 97, 299-311 (1999).

25. Gietz, R. D. \& Sugino, A. New yeast-Escherichia coli shuttle vectors constructed with in vitro mutagenized yeast genes lacking six-base pair restriction sites. Gene 74, 527-534 (1988).

26. Aparicio, O. M., Geisberg, J. V. \& Struhl, K. in Current Protocols in Molecular Biology (eds Ausubel, F. A. et al.) 21.3.1-21.3.17 (John Wiley \& Sons, New York, 2004).

27. Mogtaderi, Z. \& Struhl, K. Genome-wide occupancy of the RNA polymerase III machinery in
Saccharomyces cerevisiae reveals loci with incomplete transcription complexes. Mol. Cell. Biol. 24, 4118-4127 (2004).

28. Roth, F. P., Hughes, J. D., Estep, P. W. \& Church, G. M. Finding DNA regulatory motifs within unaligned noncoding sequences clustered by whole-genome mRNA quantitation. Nature Biotechnol. 16, 939-945 (1998)

29. Crooks, G. E., Hon, G., Chandonia, J. M. \& Brenner, S. E. WebLogo: a sequence logo generator. Genome Res. 14, 1188-1190 (2004)

30. Reid, J. L., Moqtaderi, Z. \& Struhl, K. Eaf3 regulates the global pattern of histone acetylation in Saccharomyces cerevisiae. Mol. Cell. Biol. 24, 757-764 (2004).

Supplementary Information accompanies the paper on www.nature.com/nature.

Acknowledgements We thank D. Shore for communicating similar results before publication, N. Reppas for help with computational analysis, J. Weiner for assistance with microarrays, and F. Bachand, C. Baisden, and Z. Moqtaderi for technical assistance. We thank J. Geisberg, P. Mason and Z. Moqtaderi for discussions. J.T.W. was supported by a long-term EMBO fellowship, and D.B.H. was supported by a Helen Hay Whitney postdoctoral fellowship. This work was supported by grants to K.S. from the National Institutes of Health.

Competing interests statement The authors declare that they have no competing financial interests.

Correspondence and requests for materials should be addressed to K.S.

(kevin@hms.harvard.edu).

\section{Growth-regulated recruitment of the essential yeast ribosomal protein gene activator Ifh1}

\section{Stephan B. Schawalder ${ }^{1}$, Mehdi Kabani ${ }^{2}$, Isabelle Howald ${ }^{1}$, Urmila Choudhury ${ }^{1}$, Michel Werner ${ }^{2}$ \& David Shore ${ }^{1}$}

${ }^{1}$ Department of Molecular Biology and NCCR Program 'Frontiers in Genetics' University of Geneva, Sciences III, 30, quai Ernest-Ansermet, Geneva 4, CH-1211, Switzerland

${ }^{2}$ Service de Biochimie et Génétique Moléculaire, CEA Saclay, 91191 Gif-SurYvette, Cedex, France

Regulation of ribosome biogenesis is central to the control of cell growth $^{1}$. In rapidly growing yeast cells, ribosomal protein (RP) genes account for approximately one-half of all polymerase II transcription-initiation events ${ }^{1}$, yet these genes are markedly and coordinately downregulated in response to a number of environmental stress conditions ${ }^{2-4}$, or during the transition from fermentation to respiration ${ }^{5}$. Although several conserved signalling pathways (TOR, RAS/protein kinase $\mathrm{A}$ and protein kinase $\mathrm{C}$ ) impinge upon RP gene transcription ${ }^{1}$, little is known about how initiation at these genes is controlled. Rap1 (refs 6, 7) and more recently Fhl1 (ref. 8) were shown to bind upstream of many RP genes. Here we show that the essential protein Ifh1 binds to and activates many RP gene promoters under optimal growth conditions in Saccharomyces cerevisiae. Ifh 1 is recruited to RP gene promoters through the forkhead-associated domain of Fhl1. Ifh1 binding decreases when RP genes are downregulated either by TOR inhibition or nutrient depletion, and is restored after release from starvation or upon regulated induction of IFHI expression. These findings indicate a central role for Ifh 1 and Fhl1 in RP gene regulation.

A recent global chromatin location analysis in yeast uncovered the forkhead-like protein Fhl1 as a factor highly specific for RP gene promoters $^{8}$. Fhl1 was originally identified as a gene dosage suppressor of RNA polymerase (Pol) III mutations required for normal growth ${ }^{9}$. The slow growth of fhll $\Delta$ cells is suppressed by elevated gene dosages of IFH1, suggesting that both Fhll and Ifh1 are involved in ribosomal RNA transcription or maturation ${ }^{10}$. In an attempt to resolve these apparently conflicting findings, we first 
tested the prediction ${ }^{8}$ that Fhl1 is required for normal expression of the Pol-II-driven RP genes. We examined two representative genes (RPL9A and RPL30) in fhl1 $\Delta$ cells and found reduced levels of both transcripts relative to actin (ACT1), which does not bind Fhl1 (ref. 8; see also Supplementary Table 1), suggesting that Fhl1 binding to RP gene upstream regulatory sequences (URS) is required for full expression (Fig. 1a). Similar results were seen in fhll $\Delta$ ifh $1 \Delta$ cells (Fig. 1a), and may explain why both fhll $\Delta$ and fhl1 $\Delta$ ifh $1 \Delta$ strains grow extremely slowly compared with wild type (ref. 10 and data not shown).

We next used chromatin immunoprecipitation (ChIP) to test the idea that the essential Ifh1 protein is also involved in RP gene transcription, and observed specific binding to three different RP gene URS (RPL30, RPL37A and RPL9) under optimal growth conditions (Fig. 1b). Similarly, we confirmed that both Rap1 (ref. 6) and Fhl1 (ref. 8) also bind to these URS (Fig. 1b, c). Notably, we found that Ifh 1 binding to these genes is undetectable in fhl1 $\Delta$ cells (Fig. 1b), suggesting that Fhll has an essential role in the recruitment of Ifh1 to RP genes. This effect is specific to Ifh1, because neither fhll $\Delta$ nor the fhll $\Delta$ ifh $1 \Delta$ double mutant shows any loss of Rap1 binding (Fig. 1c). Conversely, a non-RP gene bound by Rap1 (PGK1) shows no binding of either Fhl1 or Ifh1 (Fig. 1c), indicating that Rap1 binding itself does not lead to either Fhl1 or Ifh1 promoter association.

As an exponentially growing yeast culture begins to deplete glucose from the medium, cells initiate a global re-programming of transcription in preparation for the metabolic transition from fermentation to respiration ${ }^{11}$. Well before this 'diauxic shift', a marked drop in RP gene transcript levels is observed ${ }^{5}$, the mechanism of which is unknown. To address this issue we used ChIP to measure association of Rap1, Fhl1 and Ifh1 at three different RP gene promoters as cells prepared for the diauxic shift. We observed a marked decline in Ifh 1 promoter binding at RPL30 (and the two other RP genes tested; data not shown) that coincided with, or immediately preceded, the observed drop in messenger RNA level (Fig. 1d). In contrast, promoter association of Rap1 and Fhl1 remains constant during this period. The protein levels of all three factors are largely unchanged during the period of RP gene repression (Supplementary Fig. S1a). These results establish a tight temporal correlation between loss of Ifh1 promoter association and downregulation of RP gene transcription in a wild-type setting, and suggest that controlled promoter association of Ifh1 may be a key regulatory mechanism for RP genes. We also examined the RP gene association of the three proteins as stationary phase cells resume exponential growth after inoculation into fresh medium. As expected, RPL30 transcripts accumulate rapidly (approximately sevenfold increase by $15 \mathrm{~min}$; Supplementary Fig. S1b). Notably, at this early time point we observed a roughly threefold increase in Ifh1 URS association, whereas both Fhl1 and Rap1 association remained constant. These data reinforce the notion that Ifh1 binding at RP genes is associated with (and possibly required for) full activation of these genes (see below).

To investigate whether reversible Ifh 1 binding is more generally associated with RP gene regulation, we examined the response to rapamycin treatment, which inhibits TOR, a highly conserved phosphatidylinositol-kinase-like growth regulator in yeast ${ }^{12}$. As previously reported ${ }^{13-15}$, we found that rapamycin causes a rapid drop in the mRNA levels of RP genes (Fig. 1e). Notably, Ifh1 association with three different RP gene promoters begins to drop by the earliest time point tested ( $5 \mathrm{~min}$ after rapamycin addition), and is tenfold lower compared with untreated cells by $30 \mathrm{~min}$. This drop in Ifh 1 promoter association precedes any measurable change in RP gene mRNA levels (perhaps by as much as $10 \mathrm{~min}$ ), suggesting that it may be a cause of transcriptional downregulation. Notably,

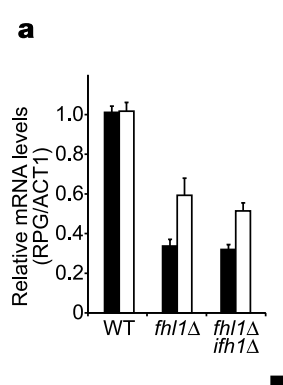

b
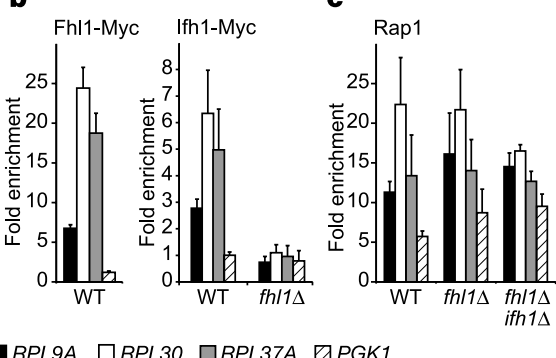

d

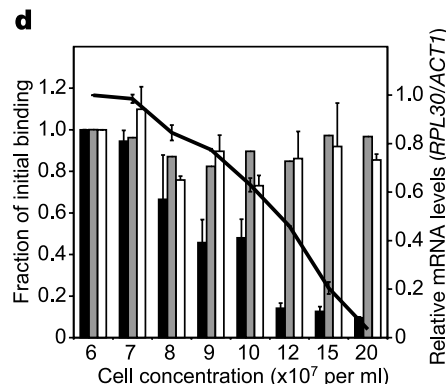

Ifh1-Myc

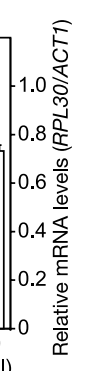

$\square$ Fhl1-Myc

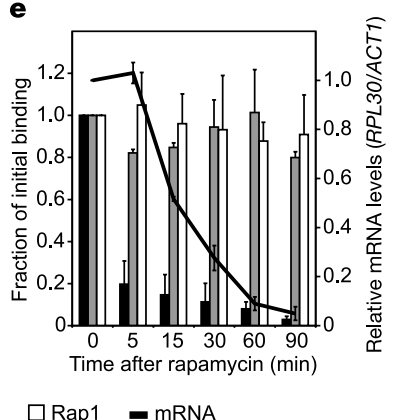

Figure 1 Ifh1, Fhl1 and Rap1 binding at RP gene (RPG) promoters, and mRNA levels during logarithmic growth, before the diauxic shift or after TOR inhibition. a, RPL9A and $R P L 30$ transcript levels relative to actin in wild-type (WT), fh/1 $\Delta$ and fh/1 $\Delta$ ifh $1 \Delta$ strains (logarithmic growth: $2 \times 10^{7}$ cells ml ${ }^{-1}$ ). b. Promoter association of Fhl1- and Ifh1-Myc at three RP gene promoters and the glycolytic gene PGK1 in logarithmic wild-type and fhl1 $\Delta$ strains. Mean values and standard deviations (s.d.) of three independent experiments are shown. c, Binding of Rap1 in wild-type, fh/1 $\Delta$ and fh/1 $\Delta$ ifh $1 \Delta$ strains, as in b. d, Diauxic shift. Fhl1- or Ifh1-Myc strains were processed for ChIP and mRNA analysis at the indicated cell concentrations (bottom). Binding for each factor at the initial time point (fold enrichment; see b, c) was set to 1. Three independent ChIP reactions and mRNA measurements for each point were performed (mean values and s.d. for one such experiment are shown). e, TOR inhibition. Fhl1- or Ifh1-Myc strains were grown to $2 \times 10^{7}$ per $\mathrm{ml}$, when rapamycin was added to $200 \mathrm{ng} \mathrm{ml}^{-1}(t=0)$. Preparation and analysis of samples was as in $\mathbf{d}$, and data shown are the average and s.d. of three independent experiments. 
and like the diauxic shift, neither Rap1 nor Fhl1 promoter binding appear to change after TOR inhibition, and total Ifh 1 protein levels are unchanged at the time that the protein disappears from the RP gene promoters (Supplementary Fig. S1a).

To determine whether Fhl1 is directly involved in recruiting Ifh1 to RP genes, we fused parts of Fhl1 to the Gal4 DNA-binding domain (GBD). We then asked whether tethering of these GBDFhl1 hybrids (Fig. 2a) to a promoter containing Gal4-binding sites, where Fhl1 is normally not bound, would be sufficient to recruit Ifh 1 there. ChIP analysis showed that this is indeed the case (Fig. 2b). Ifh1 binding at the GAL7 promoter in cells expressing GBD-Fhl1 hybrids is quantitatively similar to that observed at native RP gene promoters (data not shown), suggesting that Fhll is sufficient for robust Ifh1 recruitment at an ectopic site. Significantly, we found that the forkhead-associated (FHA) domain of Fhll, and perhaps a small amount of flanking sequence, is sufficient for Ifh1 recruitment, whereas all hybrids tested that lack this domain fail to recruit Ifh1. Furthermore, GBD-Fhl1-mediated Ifh1 recruitment is sensitive to rapamycin, suggesting that a primary downstream effect of TOR inhibition may be to interfere with the ability of Ifh 1 to associate with Fhll at RP gene promoters. Finally, although recruitment of Ifh 1 is consistently associated with activation of the GAL7-lacZ reporter (and lack of recruitment is associated with no activation) there is no strict quantitative relationship between the amount of Ifh 1 binding and the extent of activation (Fig. 2c). The carboxy terminus of Fhll, and to a lesser extent the FH domain, appear to have a repressive function in this context.

To ask whether the Fhl1-Ifh1 interactions described above at three RP gene promoters are common to most, or all, RP genes, we conducted a chromosomal binding analysis of both Ifh 1 and Fhl 1 through hybridization of immunoprecipitated DNA to yeast intergenic microarrays ${ }^{6,16,17}$. As reported previously ${ }^{8}$, we found that Fhl chromatin binding in vivo is highly specific for RP genes. We found the same to be true for Ifh1, the chromosomal binding sites of which remarkably overlap those found for Fhl1 (Supplementary Fig. S2a, b). Fhl1 and Ifh1 bound to $53 \%$ (72) and to $45 \%$ (61) $(P<0.001)$ of the $137 \mathrm{RP}$ gene promoters, respectively. Moreover, $34 \%$ (46) of RP gene promoters were bound by both factors. Owing to the stringency of our analysis and technical limitations, we believe that these data actually underestimate the co-localization of these factors. We repeated our analysis with cells that had been

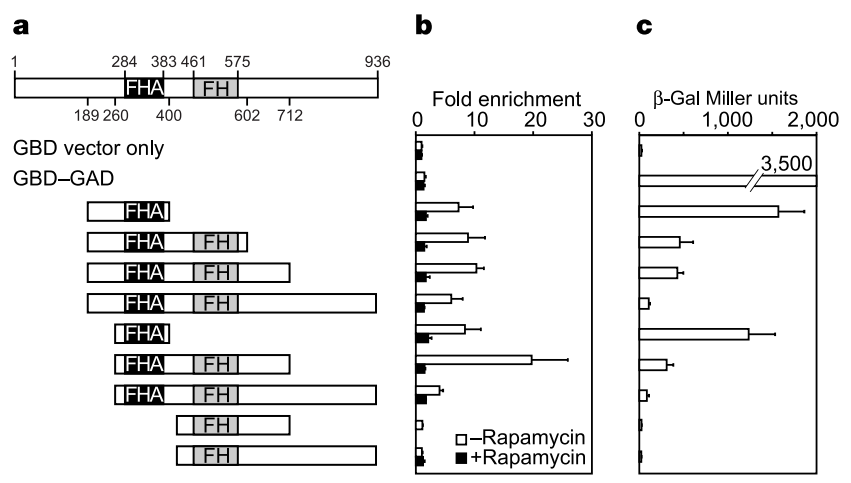

Figure 2 Rapamycin-sensitive recruitment of Ifh1 to an ectopic site (GAL7) via the FHA domain of GBD-Fhl1 hybrids. a, Schematic representation of native Fhl1 (ref. 28) and GBD-Fhl1 hybrid proteins. Numbers above the bar indicate amino acid positions of domain boundaries; those below indicate the fusion endpoints of GBD-Fhl1 hybrids. b, Ifh1-Myc binding at GAL7 as measured by ChIP in cells growing exponentially or 90 min after rapamycin treatment. Cells express control (GDB alone or GBD-GAD) or GBD-Fhl1 hybrid proteins, as indicated in $\mathbf{a}$. The mean values and s.d. of three independent experiments are shown. $\mathbf{c}, \beta$-Galactosidase activity in cells expressing the proteins indicated in $\mathbf{a}$ treated with rapamycin before crosslinking, and found a markedly different result for Ifh1 binding, as predicted by our study of three specific RP genes. Specifically, rapamycin treatment reduced binding to background levels at all but one RP gene, and no new sites of Ifh1 binding were identified (Supplementary Fig. S2 and Supplementary Table 1). As expected from our initial studies, we observed very little effect of rapamycin on global Fhl1 binding.

We next asked whether Ifh1 functions as a direct transcriptional activator of native RP genes, as the above results strongly imply. To address this question we constructed a strain in which the endogenous IFH1 gene is controlled by the galactose-inducible GAL1 promoter. This strain grows slowly and contains very low levels of Ifh1 protein on medium containing glucose (which represses
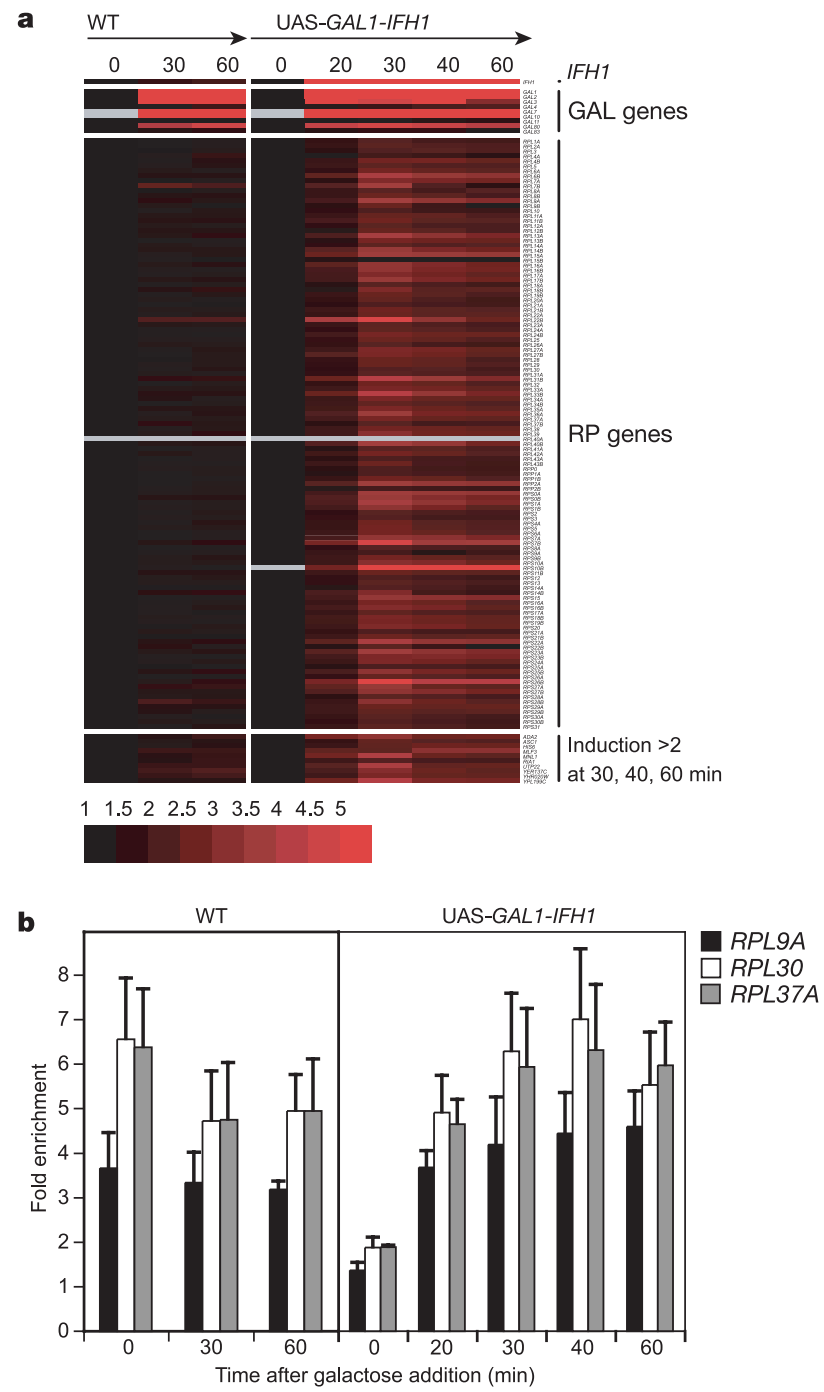

Figure 3 Ifh1 directly activates RP gene transcription. Isogenic wild-type and UAS-GAL1IFH1 cells were grown to $2 \times 10^{7}$ cells ml $^{-1}$ in YPLG medium (containing lactic acid and glycerol) and $t=0$ samples collected. Galactose was then added to $2 \%$ and samples collected at the indicated time points (in minutes). a, mRNA expression ratios of induced genes relative to the initial YPLG culture $(t=0)$, as measured by microarray analysis, are shown for each time point. Genes specifically induced in UAS-GAL1-IFH1 cells (>twofold) at $t=30,40$ and 60 were grouped. Red indicates $>$ onefold increase (induction) and grey signifies that no reliable measurement was obtained. Functional classes of genes are indicated, except from the ten genes grouped at the bottom (see text and Supplementary Fig. S3 for details). b, Ifh-Myc binding to three RP gene promoters (RPL9A, RPL30 and $R P L 37 A)$ for each time point as measured by ChIP. The mean values and s.d. of three independent experiments are shown. See Supplementary Information for further details. 
UAS-GAL1-IFH1), but grows normally on galactose (inducing) medium (data not shown). We examined, by genome-wide microarray analysis, the effect of inducing IFH1 expression in this strain by shifting cells from non-inducing (lactic acid and glycerol) to inducing medium. Nearly all mRNAs of RP genes were detectably upregulated only $20 \mathrm{~min}$ after galactose addition, and by $30 \mathrm{~min}$ were typically two- to fivefold induced, in all cases exceeding the modest induction observed for some RP genes in the control (IFH1 wild type) strain (Fig. 3a). In the three cases examined directly by ChIP, this increase in RP gene transcript was associated with increased Ifh1 promoter binding, again unlike the control strain where Ifh1 binding remained constant (Fig. 3b). Because the RP genes were among the primary targets after induction of Ifh 1 , we conclude that Ifh1 is a direct transcriptional activator of these genes. Only ten other Pol II protein-encoding genes were induced as rapidly and as strongly as the RP genes (Fig. 3a). Among these genes UTP22, ASC1 and RIA1 are bound by both Rap1 and Fhl1 (ref. 8; see also Supplementary Fig. S2) and are involved in ribosome biogenesis. Our data thus point to a possible role for Ifh1 in coordinating RP gene activation with other key steps in ribosome biogenesis.

The results reported here support a simple model in which normal RP gene expression under favourable growth conditions requires recruitment of the essential (co)activator Ifh1 to RP gene promoters through an interaction with the FHA domain of promoter-bound Fhl1. This model shares several features with that proposed to explain the action of two other FH proteins, Fkh1 and Fkh2, together with the essential protein Ndd1 in activation of the 'CLB2 cluster' of G2/M-specific genes ${ }^{18,19}$. One curious property of both systems is that the requirement of the co-activator for cell growth (Ifh1 here; Ndd1 for the Fkh1/2 system) can be bypassed by deletion of the DNA-binding recruitment factor ${ }^{10}$. In cells lacking Ifh1, Fhl1 may repress RP gene transcription, either alone or with a co-repressor, and thus block cell growth. In the absence of both proteins, activation of RP genes is low but sufficient to permit cell growth.

Our data also imply that the Fhl1-Ifh1 interaction constitutes a major regulatory node in the system: both glucose (possibly through the RAS/protein kinase A pathway) and TOR activity are required for full recruitment of Ifh 1 to RP gene promoters. Significantly, inactivation of either one of these two major growth stimulatory pathways results in a rapid loss of Ifh1 promoter binding, which is followed by a large drop in RP gene mRNA levels. The mechanism(s) by which this occurs are unknown. The fact that Ifh1 is recruited by the FHA domain of Fhl1 (Fig. 2b), and that FHA domains in various other proteins are phospho-peptide-binding motifs ${ }^{20}$, suggests that Ifh 1 recruitment at RP gene promoters may be sustained by active, reversible phosphorylation of a putative Fhl1-binding domain in Ifh1. Whether this occurs through the direct action of protein kinase A, TOR, or both kinases, is at present unknown.

The findings reported here do not exclude the possibility that other important control mechanisms act directly at RP gene promoters. Indeed, the coordinated downregulation of RP genes under a wide range of stress conditions, and the importance to the cell of this regulation from an energetic standpoint ${ }^{1}$, suggests that multiple mechanisms are probably involved. An obvious additional candidate for regulatory input at RP gene promoters is Rap1 (ref. 7, 21 ), which could function in promoting proper Fhll binding, or in the recruitment of the Esal histone acetyltransferase and/or the TAF-containing form of TFIID ${ }^{22-24}$. Finally, the recently identified growth regulator Sfp1 is an additional candidate regulator of RP genes $^{25-27}$. An understanding of RP gene regulation will provide a detailed picture of how the yeast cell integrates various growth signals at the level of the promoter. This knowledge should help to dissect further the signal pathways that regulate ribosome biogenesis and growth in yeast, and is likely to have important implications in more complex multicellular organisms.

Received 30 September; accepted 18 November 2004; doi:10.1038/nature03200.

1. Warner, J. R. The economics of ribosome biosynthesis in yeast. Trends Biochem. Sci. 24, 437-440 (1999).

2. Gasch, A. P. et al. Genomic expression programs in the response of yeast cells to environmental changes. Mol. Biol. Cell 11, 4241-4257 (2000).

3. Causton, H. C. et al. Remodeling of yeast genome expression in response to environmental changes. Mol. Biol. Cell 12, 323-337 (2001).

4. Rep, M., Krantz, M., Thevelein, J. M. \& Hohmann, S. The transcriptional response of Saccharomyces cerevisiae to osmotic shock. Hotlp and Msn2p/Msn4p are required for the induction of subsets of high osmolarity glycerol pathway-dependent genes. J. Biol. Chem. 275, 8290-8300 (2000).

5. Ju, Q. \& Warner, J. R. Ribosome synthesis during the growth cycle of Saccharomyces cerevisiae. Yeast 10, 151-157 (1994)

6. Lieb, J. D., Liu, X., Botstein, D. \& Brown, P. O. Promoter-specific binding of Rap1 revealed by genomewide maps of protein-DNA association. Nature Genet. 28, 327-334 (2001).

7. Shore, D. RAP1: a protean regulator in yeast. Trends Genet. 10, 408-412 (1994).

8. Lee, T. I. et al. Transcriptional regulatory networks in Saccharomyces cerevisiae. Science 298, 799-804 (2002)

9. Hermann-Le Denmat, S., Werner, M., Sentenac, A. \& Thuriaux, P. Suppression of yeast RNA polymerase III mutations by FHL1, a gene coding for a fork head protein involved in rRNA processing. Mol. Cell. Biol. 14, 2905-2913 (1994).

10. Cherel, I. \& Thuriaux, P. The IFH1 gene product interacts with a fork head protein in Saccharomyces cerevisiae. Yeast 11, 261-270 (1995).

11. DeRisi, J. L., Iyer, V. R. \& Brown, P. O. Exploring the metabolic and genetic control of gene expression on a genomic scale. Science 278, 680-686 (1997).

12. Jacinto, E. \& Hall, M. N. Tor signalling in bugs, brain and brawn. Nature Rev. Mol. Cell Biol. 4, 117-126 (2003)

13. Cardenas, M. E., Cutler, N. S., Lorenz, M. C., Di Como, C. J. \& Heitman, J. The TOR signaling cascade regulates gene expression in response to nutrients. Genes Dev. 13, 3271-3279 (1999).

14. Hardwick, J. S., Kuruvilla, F. G., Tong, J. K., Shamji, A. F. \& Schreiber, S. L. Rapamycin-modulated transcription defines the subset of nutrient-sensitive signaling pathways directly controlled by the Tor proteins. Proc. Natl Acad. Sci. USA 96, 14866-14870 (1999).

15. Powers, T. \& Walter, P. Regulation of ribosome biogenesis by the rapamycin-sensitive TOR-signaling pathway in Saccharomyces cerevisiae. Mol. Biol. Cell 10, 987-1000 (1999).

16. Ren, B. et al. Genome-wide location and function of DNA binding proteins. Science 290, 2306-2309 (2000)

17. Harismendy, O. et al. Genome-wide location of yeast RNA polymerase III transcription machinery. EMBO J. 22, 4738-4747 (2003).

18. Koranda, M., Schleiffer, A., Endler, L. \& Ammerer, G. Forkhead-like transcription factors recruit Ndd1 to the chromatin of G2/M-specific promoters. Nature 406, 94-98 (2000).

19. Jorgensen, P. \& Tyers, M. The fork'ed path to mitosis. Genome Biol. 1, R1022.1-1022.4 (2000).

20. Durocher, D. \& Jackson, S. P. The FHA domain. FEBS Lett. 513, 58-66 (2002).

21. Klein, C. \& Struhl, K. Protein kinase A mediates growth-regulated expression of yeast ribosomal protein genes by modulating RAP1 transcriptional activity. Mol. Cell. Biol. 14, 1920-1928 (1994).

22. Mencia, M., Moqtaderi, Z., Geisberg, J. V., Kuras, L. \& Struhl, K. Activator-specific recruitment of TFIID and regulation of ribosomal protein genes in yeast. Mol. Cell 9, 823-833 (2002).

23. Reid, J. L., Iyer, V. R., Brown, P. O. \& Struhl, K. Coordinate regulation of yeast ribosomal protein genes is associated with targeted recruitment of Esal histone acetylase. Mol. Cell 6, 1297-1307 (2000).

24. Rohde, J. R. \& Cardenas, M. E. The tor pathway regulates gene expression by linking nutrient sensing to histone acetylation. Mol. Cell. Biol. 23, 629-635 (2003).

25. Jorgensen, P., Nishikawa, J. L., Breitkreutz, B. J. \& Tyers, M. Systematic identification of pathways that couple cell growth and division in yeast. Science 297, 395-400 (2002)

26. Marion, R. M. et al. Sfpl is a stress- and nutrient-sensitive regulator of ribosomal protein gene expression. Proc. Natl Acad. Sci. USA 101, 14315-14322 (2004).

27. Jorgensen, P. et al. A dynamic transcriptional network communicates growth potential to ribosome synthesis and critical cell size. Genes Dev. 18, 2491-2505 (2004).

28. Kim, M., Ahn, J. W., Song, K., Paek, K. H. \& Pai, H. S. Forkhead-associated domains of the tobacco $\mathrm{NtFHAl}$ transcription activator and the yeast Fhll forkhead transcription factor are functionally conserved. J. Biol. Chem. 277, 38781-38790 (2002)

\section{Supplementary Information accompanies the paper on www.nature.com/nature.}

Acknowledgements We thank J. Warner, E. Di Mauro, K. Mizuta, G. Ammerer, P. Thuriaux and M. Tyers for discussions; J. Warner, K. Struhl and M. Tyers for communicating unpublished results; A. Bianchi for comments on the manuscript; members of the Shore laboratory for discussions; P. Descombes, C. Barraclough, C. Delucinge, O. Schaad, P. Soularue and X. Gidrol for help with microarray experiments and analysis; S. Gasser for providing antibodies; and N. Roggli for artwork. This work was supported by the Human Frontier Science Program, the Swiss National Science Foundation, the NCCR program 'Frontiers in Genetics' (Swiss National Science Foundation), and the Canton of Geneva.

Competing interests statement The authors declare that they have no competing financial interests.

Correspondence and requests for materials should be addressed to D.S.

(David.Shore@molbio.unige.ch). 